\title{
CREATING UBIQUITOUS INTERACTIVE GAMES USING EVERYWHERE DISPLAYS PROJECTORS
}

\author{
Claudio Pinhanez \\ IBM Research, T.J. Watson \\ pinhanez@us.ibm.com
}

\begin{abstract}
This paper introduces the Everywhere Displays projector, a device that uses a rotating mirror to steer the light from an LCD projector onto different surfaces of an environment and a pan/tilt camera to sense interaction with the projected surface. The ED-projector is a generic I/O ubiquitous device that is able to create graphical interfaces on non-wired surfaces, to access computational power and networked data. Also, ED-projectors can be used to augment reality without the use of goggles. In both cases, ED-projectors can be used to create a new generation of games that are played by one or more users on the real world, without requiring them to wear goggles neither asking for specially wired surfaces or objects. Some ideas of the games that can be created with the ED-projector are introduced and examined.
\end{abstract}

Key words: Ubiquitous games, ubiquitous computing, home entertainment

\section{Introduction}

The Everywhere Displays project at IBM Research aims to develop truly ubiquitous graphical interactive displays. In particular, we are interested in creating devices that allow the transformation of any surface in a space into a projected "touch-screen", without the need of installing LCD displays or of wiring sensors to the surface.

We are currently developing the Everywhere Displays projector, or EDprojector, a steerable projection system that deflects the image of an LCD projector using a rotating mirror and senses user interaction with a video camera. With this apparatus, it is possible not only to create computer displays on any surface of a space (see fig. 1) but also to enable the computer to directly act upon objects and people in the real world through

The original version of this chapter was revised: The copyright line was incorrect. This has been corrected. The Erratum to this chapter is available at DOI: 10.1007/978-0-387-35660-0_65 


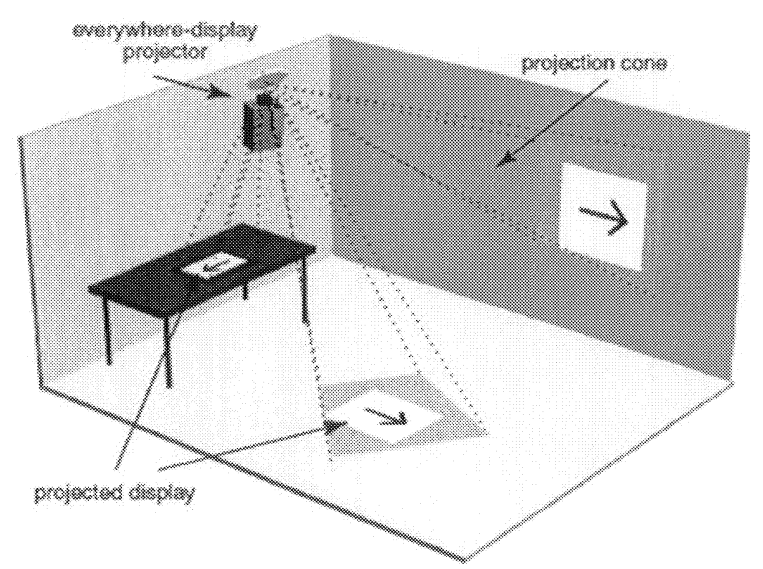

Figure 1. Using the Everywhere Displays projector to create displays on different surfaces in an environment.

the projection of images and light patterns. We have envisioned many applications for the ED-projector, from pervasive access to computer networks to systems that guide people in hallways as described later in this paper (see also [4]).

In addition, we believe that this technology can have a tremendous impact on computer games. Traditionally, computer games are played on a screen and force the participants to enter the virtual world of the computer. Although there has been recently an increasing interest on games based on PDAs and cellular phones, these devices have very poor rendering capabilities, yielding games highly based on text, similar to the first on-line games such as "Dungeons and Dragons".

One of the chief characteristics of the ED-projector is its ability to augment reality by projecting high-resolution graphical information directly on objects of the real world without requiring any change on their surfaces. In many ways, the ED-projector exploits the many surfaces already available in our environment to made them act as the placeholders of information.

This paper describes briefly the Everywhere Displays projector, its technology, and some identified applications. We follow by identifying and exploring possible uses of the ED-projector in games and education and conclude by examining the required steps to evolve its underlying technology.

\section{The Everywhere Displays Projector}

The Everywhere Displays projector, or ED-projector, is composed of an LCD projector, a computer-controlled pan/tilt mirror, and a pan/tilt/zoom 
camera. As illustrated in fig. 1, when the projection is not orthogonal to the surface the projected image can appear distorted. To correct the distortions caused by oblique projection, the image to be projected must be inversely distorted prior to projection. To accomplish this, our system first texturemaps the image onto a virtual computer graphics 3D surface that corrects the distortion. Of course, the virtual 3D surface must be uniquely determined for each surface where images are projected (see [4]).

Although a device that simply projects images on multiple surfaces has many applications, we are particularly interested in making the projected surface interactive like a touch screen. We are currently employing a pan/tilt video camera that is directed by the computer so it has a complete view of the projected surface. The user can interact by moving her hand over the projected surface, as if the hand was a computer mouse; and by moving the hand towards the surface, to generate a "click" event. The computer vision system is described in [3].

\subsection{Applications}

The ED-projector is a generic input/output device with multiple applications. A first class of applications corresponds to the creation of computer desktop-like interactive displays on non-tethered surfaces. For example, in an office, a desktop application can be projected directly on a whiteboard. Unlike the interactive whiteboard described in [2], the projected application can be moved around the room; for instance, to the top of a desk for more detailed reading.

Similarly, the ED-projector can be used to bring information to the physical location where it is used or needed. Figure 2.1 shows a situation where an electronic phone directory is projected close to a telephone. Figure 2.2 shows a database application accessing a list of files is projected on the top of the file cabinet that contains those files. When looking for a particular file, the user can access and/or update information about the file.

A second class of applications deals with the provision of means for the computer to act on the physical world, almost like a robotic arm made of light. These applications can use the ED-projector to point to physical objects, show connections among them, and project patterns to indicate movement or change in the real world.

Figure 2.3 shows an example where the ED-projector responds a request for the position of an object (a digital camera) by directly pointing to the location of the object in the room. Figure 2.4 shows another example of spatial interaction where the ED-projector is used to project a notification of an e-mail arrival on the wall. In Figure 2.5 the projector is used to signal the 

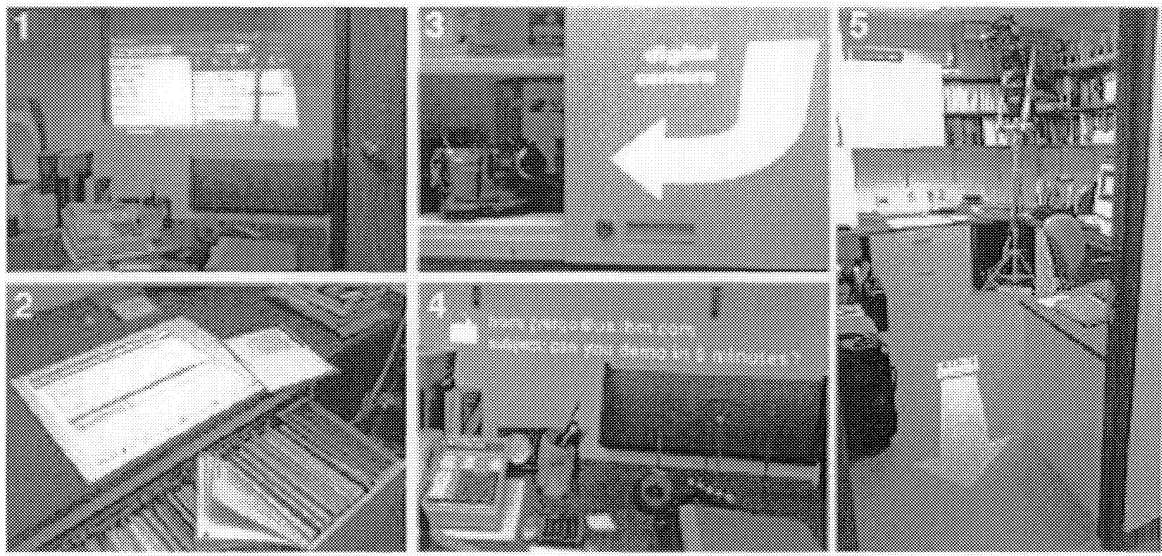

Figure 2. Applications of Everywhere Displays: (1) an electronic phone directory is projected close to the phone; (2) the database corresponding to a file cabinet is accessed directly from the top of the cabinet; (3) a localization system points to the position of an object; (4) a notification of the arrival of an urgent e-mail is silently projected on the wall; (5) an emergency sign projected on the floor directly points to the nearest exit.

direction of an emergency exit. Notice that the rotating mirror allows one single projector to switch among any of these different tasks as needed.

The ED-projector can also be used to provide computer and information access in spaces where traditional displays can be broken or stolen, or create hazardous conditions, such as in public spaces and areas subject to harsh environmental conditions. The device also permits an interactive display to be brought to the proximity of a user without requiring the user to move. In particular, the ED-projector can facilitate the access and use of computers by people with locomotive disabilities. For instance, it can project an interactive display on the sheet of a hospital bed without creating the risk of patient contact with any device.

\section{ED-Projectors in Ubiquitous Games}

In the last decade arcade computer games have increasingly explored physical elements to increase the level of user immersion. Among many examples we can cite the case of "Dance, Dance, Revolution," an arcade game extremely popular in Japan where the players have to hit spots on the floor following the rhythm of the music. However, almost all home consolebased computer games are still played on worlds locked behind the glass of computer and TV screens.

We see the Everywhere Displays projector as a potential enabler of a new generation of games that happen not in the virtual world but in the real of the physical, everyday world where we live. Unlike games based on phones and 
portable computers [1], the use of the ED-projector provides high-resolution displays where characters and fantastic objects can be created. Also, they can move to different surfaces of an environment to create a game that surrounds the players. Coupled with the camera input, the users' interaction can be collected without contact with special devices and in a modality that suits the game needs: hand gestures, body movements, foot action, and/or facial expressions. Above all, an ED-projector installed on the ceiling of a living room (or arcade environment) can be completely reconfigured just by software to create completely different games.

\subsection{Entertainment}

Let's imagine an ED-projector installed on the ceiling of a children's bedroom. First, any surface of the room is now potentially a display, so "Age of Empires ${ }^{\otimes}$ " or "SimCity" can magically happen on the floor, or on the children's bed. Interaction with the characters is accomplished by directly moving the hand over the projected surface, or by using companion plastic tokens recognizable by the camera.

Another possibility is to use the ED-projector to enhance traditional floor-based games such as hopscotch. The size and difficulty of the game can be adjusted to different ages easily. Also, players in different geographic locations can share the same game, and follow each other by watching footprints representing their hops.

Another possibility is to use the ED-projector to populate an environment with characters or creatures. For instance, different faces can be projected on drawers, transforming each of the drawers into a character. Unlike the case of "The KidsRoom" and similar environments [5], no complex and sitespecific installation is required. Alternatively, a game can explore the objects and furniture present in the room to hide characters and objects. For example, we can imagine a game where ants are invading the house and the players' task is to find and drive them away. The players may have to look ants that are hid den in closets, under the bed or the carpet, or simply having a feast inside the trashcan.

In many ways, the main constrain to implement such games comes, in fact, from the sensing mechanisms. Although computer vision techniques work in many special cases, it will require some time to construct systems that automatically recognize and track beds and trashcans. However, unlike other applications, games allow for setup time where the users may teach the system where the needed objects are, how the players look like, etc. 


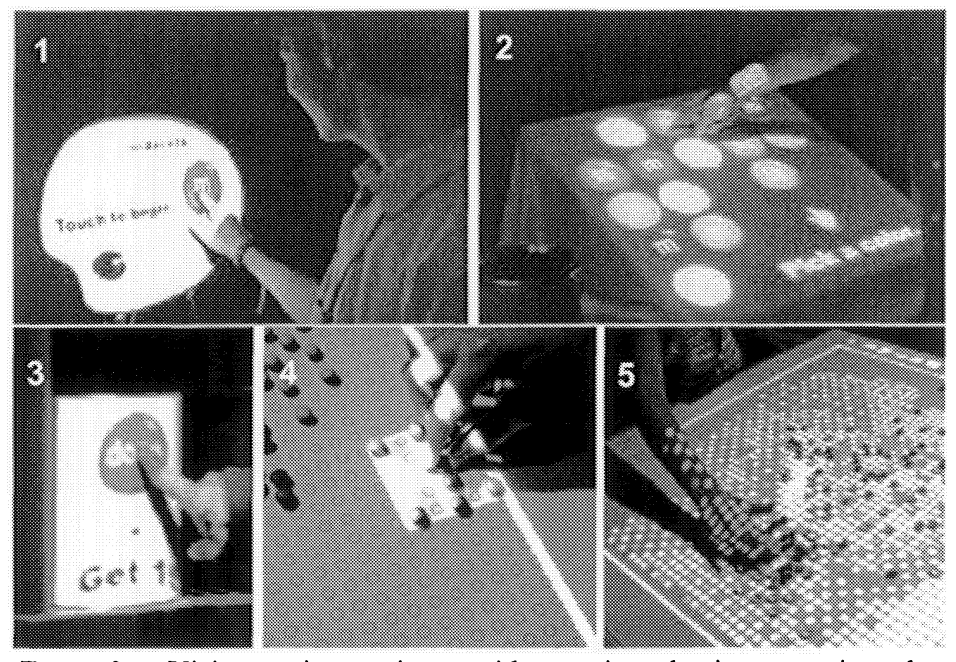

Figure 3. Visitors interacting with projected images in the SIGGRAPH'01 demo: (1) the entrance panel is touched to start the process; (2) a color is selected; (3) the bucket is "clicked" after the M\&M's ${ }^{\circledR}$ are picked up; (4) the user places the M\&M's ${ }^{\circledR}$ on the sticky surface; (5) finger-painting to reveal the full image.

\subsection{A Game-Like Experience with M\&Ms}

At the Emerging Technologies venue of SIGGRAPH'01 we created a game-like installation that demonstrated some of the possible applications of ED-projectors to games. First of all, the goal of this experiment was not to create a game, but instead to create an experience where people could be introduced to the ED-projector concept and technology.

However, given the characteristics and interests of our audience, we designed an installation where people collaboratively build an object, piece by piece as in a puzzle. The object to be assembled is a picture made of M\&M's ${ }^{\circledast}$ (multi-colored sugar-coated chocolates) where each $M \& M^{\otimes}$ is regarded as a "pixel" of the picture. The pictures were $60 \times 50 \mathrm{M} \& \mathrm{M}^{\otimes}$ "pixels" and required the collaboratively work of more than 200 people during the course of several hours.

The installation was designed so the computer system would show each visitor how to contribute. Figure 3 describes some of the steps of this process. Each visitor was given the choice of which color of M\&M's ${ }^{\circledR}$ she would like to place in the picture. Then, the system pointed an unlabeled bucket containing $M \& M^{\prime} s^{\circledast}$ of that color. Following, the places where the M\&M's ${ }^{\circledast}$ should be placed were highlighted. And finally, the visitor could "finger paint", that is, wave her hand on top of the placement board to reveal 

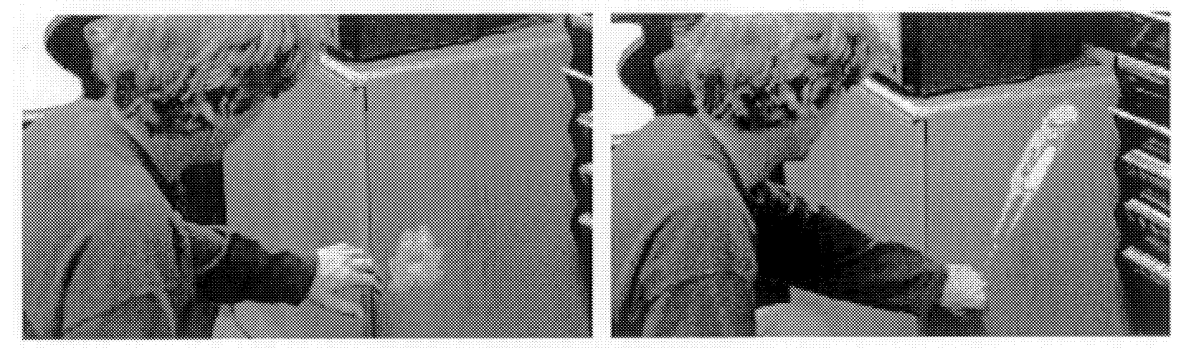

Figure 4. Trying to catch a projected frog.

the complete picture. A detailed description of this installation is beyond the scope of this paper (see [6]).

\section{3 "Fro...og!"}

The ED-projector's unique characteristic of being able to quickly move the projected image to different surfaces is being explored in a computer game for young children. In this game, called "Fro...og!", an image of a frog is projected on a surface. Whenever a child tries to touch the frog, the camera detects her approach, and the frog "jumps" to another surface, that is, the projector moves the image to a wall or the top a chair.

Figure 4 shows two pictures of a user playing with the current prototype of "Fro...og!". The basic idea is to define surfaces in the environment where the animated frog can jump to. Whenever the user's hand approaches the frog, the next surface is randomly chosen, and an image of the frog jumping in that direction is briefly shown. After landing on the new surface, a simple animation of the frog jumping up and down is projected, so it becomes easier to find the frog in the environment.

In the next development phase of this game we plan to integrate it to a system that tracks the position of multiple children in the room, so the frog can jump to surfaces that are far from most players. Tracking is also particularly important to avoid projecting the frog directly onto the players that, in this scenario, can easily break the illusion of the game.

\section{Final Remark}

Currently we had not yet implemented any of the entertainment or educational ideas described here, except for the $M \& M^{\circledast}$ installation. Although most of our current research efforts are related to improve the technology of ED-projectors, we are greatly interested on its use in different applications, and in games and education in particular. 
A major obstacle for the introduction of ED-projectors in tomes is the calibration of the different used surfaces to compensate for oblique projection distortion. Similarly, many factors may hinder a computer vision system in a home, especially lighting conditions. Both issues are part of the main focus of our research but we should expect that progress in those fronts will not come immediately.

In the case of games, we expect that the first implementation will happen not in the realm of home, but in arcades, museums, and theme parks. It is possible, in these cases, not only to constrain the environment to suit the technological restrictions but also to count on professional setup and maintenance. Moreover, such public spaces often help gamers to acquire new interface and game concepts, mostly learned by observation of other players. Once a player has experienced the transformation of a floor into a touch screen in an arcade, it is much easier for him to accept that he can interact with a characters projected on the pillows of his bedroom.

\section{Acknowledgements}

The SIGGRAPH'01 installation was designed and implemented by Claudio Pinhanez, Rick Kjeldsen, Anthony Levas, Gopal Pingali, Jacob Hartman, Vivek Kwatra, Mark Podlaseck, and Paul Chou. We would also like to thank the support from the M\&M/Mars and the SIGGRAPH'01 Emerging Technologies Committee. M\&M is a registered trademark of M\&M Mars, Inc.

\section{References}

[1] S. Björk, et al., "Pirates! - Using the Physical World as a Game Board", Proc. of Interact'01, Tokyo, Japan, 2001.

[2] J.L. Crowley, et al., "Things that See", Communications of the ACM, 43(3): 54-64, 2000.

[3] R. Kjeldsen, et al., "Interacting with Steerable Projected Displays", Proc. of Face and Gesture'02, 2002.

[4] C. Pinhanez, "The Everywhere Displays Projector: A Device to Create Ubiquitous Graphical Interfaces", Proc. of Ubiquitous Computing 2001 (Ubicomp'01), Atlanta, Georgia, 2001.

[5] C. Pinhanez, et al, "Physically Interactive Story Environments", IBM Systems Journal, 39(3\&4), pp. 438-455, 2000.

[6] C. Pinhanez, et al., "Transforming Surfaces Into Touch-Screens", IBM Research Report RC22273 (W0112-0216), 2001. 